\title{
Design and Validation of Non-Exercise Equations for Estimation of Aerobic Capacity in Iranian Boys
}

\author{
Farzad Nazem ${ }^{1 *}$ (D), Abbas Rezaei ${ }^{1}$ (D), Majid Jalili ${ }^{1}$, Hossein Saki ${ }^{1}$ (D)
}

1. Department of Physical Education and Sport Sciences, Section of Sport Physiology, Bu-Ali Sina University, Hamadan, Iran

\begin{tabular}{|c|}
\hline Article Info \\
\hline $\begin{array}{l}\text { Received: 2019/12/24; } \\
\text { Accepted: 2020/08/17; } \\
\text { ePublished: 2020/10/15 }\end{array}$ \\
\hline doi $10.30699 /$ jergon.8.2.50 \\
\hline $\begin{array}{l}\text { Use your device to scan } \\
\text { and read the article online }\end{array}$ \\
\hline 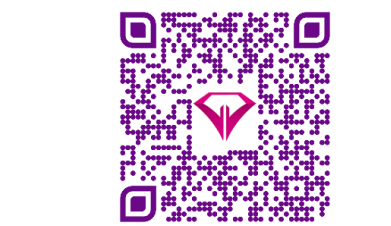 \\
\hline
\end{tabular}

Corresponding Author Farzad Nazem

Department of Physical Education and Sport Sciences, Section of Sport Physiology, BuAli Sina University, Hamadan, Iran

Email:

f.nazem1336@gmail.com

\section{ABSTRACT}

Background and Objectives: Accurate and rapid measurement of cardiorespiratory system performance by estimation methods regardless of basic physiological fitness level, geographical environment, culture and nutrition is of paramount importance. The purpose of this study was to design linear regression equations to estimate the aerobic capacity of healthy adolescent boys without the use of sport tests and validation of this equations.

Methods: In the present semi-experimental study, 156 healthy adolescent boys aged 13 to 17 years with a mean body mass index of $21.43 \pm 4.5 \mathrm{Kg} / \mathrm{m}^{2}$ were selected by purposive sampling method. To design the non-sport equation for estimating maximum oxygen consumption $\left(\mathrm{VO}_{2 \text { peak }}\right)$, a multiple regression linear model and Pearson correlation were used to validate the equations.

Results: Significant correlations were observed between the measured $\mathrm{VO}_{2 \text { peak }}$ and the anthropometric and physiological variables $(\mathrm{R}=0.122-0.799, P<0.001)$. Also, a valid non-exercise linear equation for boys' $\mathrm{VO}_{2 \text { peak }}$ prediction was designed with variables such as age, $\mathrm{BMI}$ and resting heart rate $\left(\mathrm{SEE}=3.59 \mathrm{~mL} / \mathrm{kg} / \mathrm{min}, \mathrm{R}^{2}=\right.$ $0.712, P<0.001)$. The estimated $\mathrm{VO}_{2 \text { peak }}$ from equations had a significant correlation with the obtained criterion value. $(\mathrm{R}=0.707-0.730, P<0.01)$.

Conclusion: According to the results of the present study, it is possible to use aerobic capacity estimation equations, is a simple, accurate, safe tool in assessing the baseline cardiorespiratory fitness $\left(\mathrm{VO}_{2 \text { peak }}\right)$. The use of non-Exercise equations in the planning of exercise in large populations of adolescent boys and even as a daily clinical practice in the elderly and heart patients with the goals of promoting health, cardiovascular health, preventive actions is very important.

Keywords: Aerobic capacity, Respiratory gas analysis, Non-exercise testing, Validation

Copyright (C) 2020, This is an original open-access article distributed under the terms of the Creative Commons Attribution-noncommercial 4.0 International License which permits copy and redistribute of the material just in noncommercial usages with proper citation.

How to Cite This Article:

Nazem F, Rezaei A, Jalili M, Saki H. Design and Validation of Non-Exercise Equations for Estimation of Aerobic Capacity in Iranian Boys. Iran J Ergon. 2020; 8 (2): 50-60 


\section{طراحى و اعتبارسنجى معادلات غيرورزشى برآورد ظرفيت هوازى در پِران ايرانى بـى}

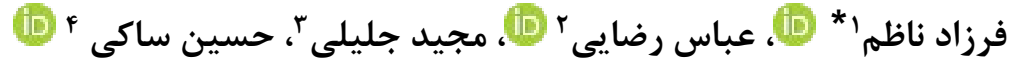

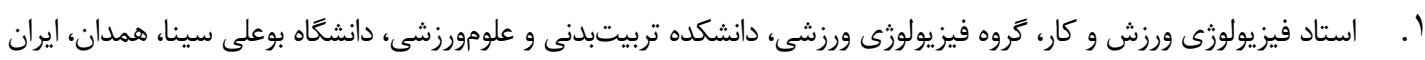

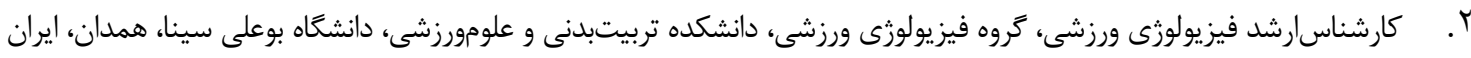

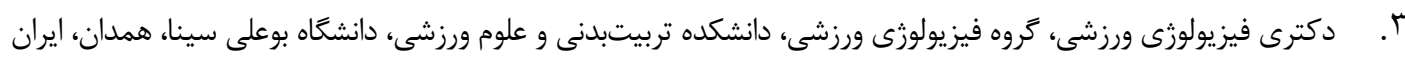

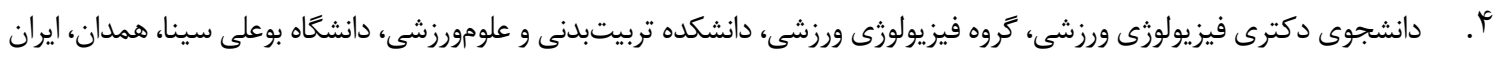

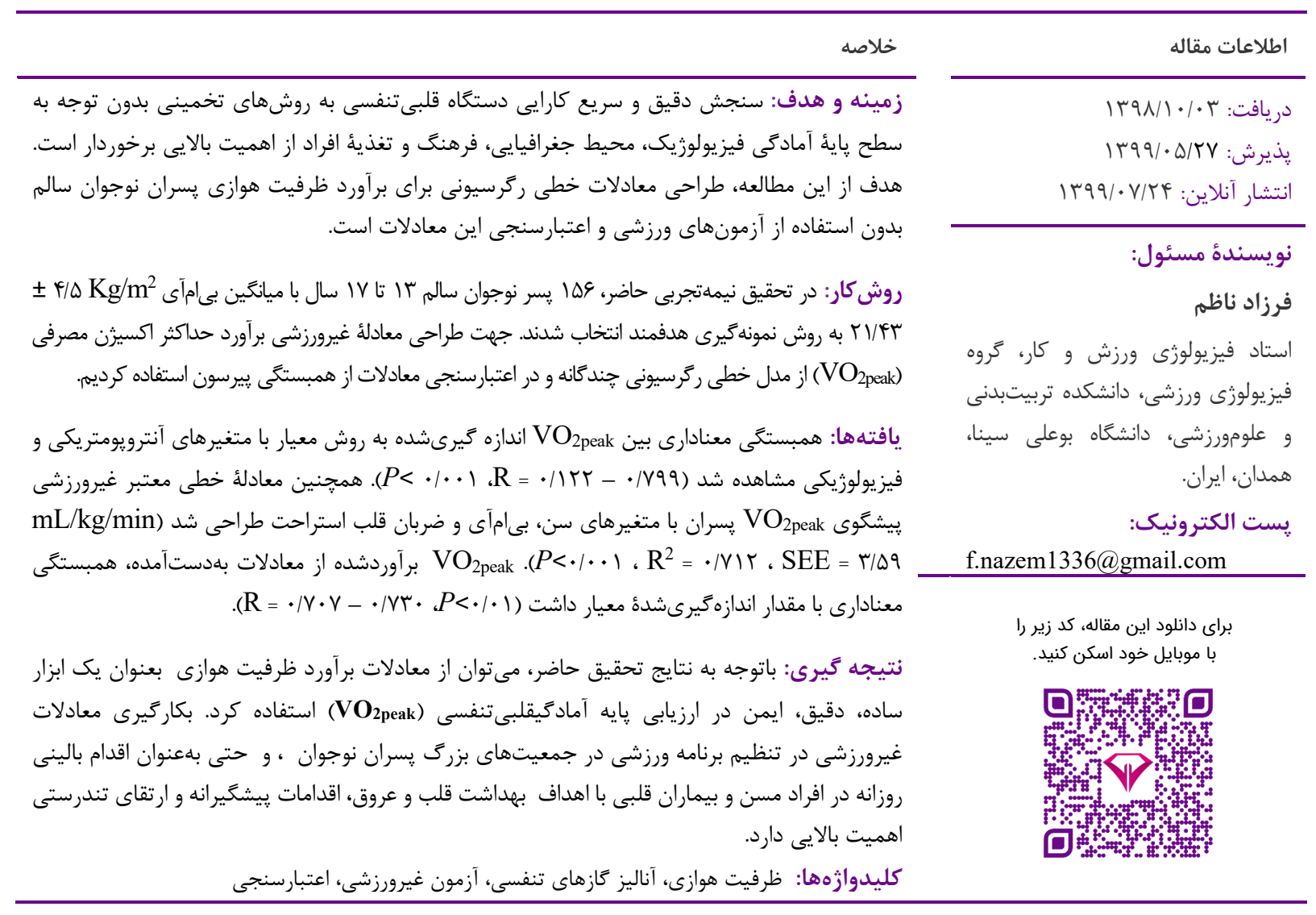

مقدمه

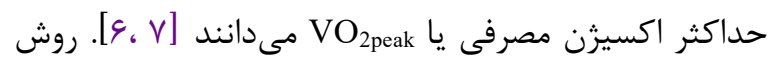
VO استاندارد ارزيابى CRF اندازمكيرى مستقيم

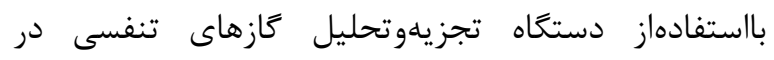

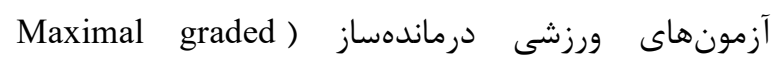
(exercise test

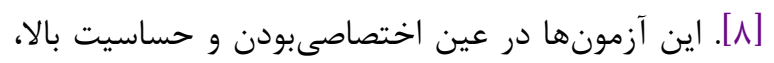

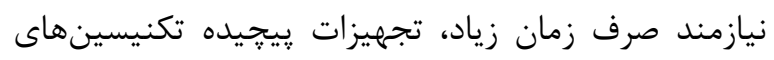

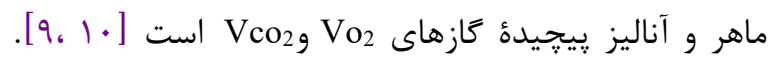

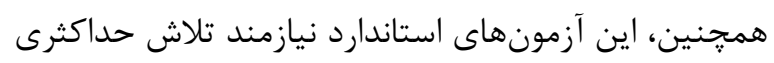

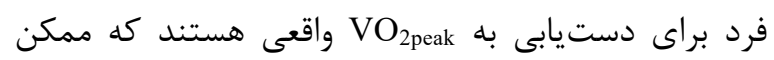

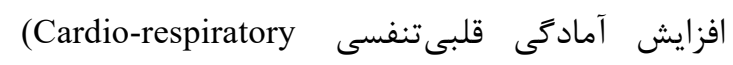

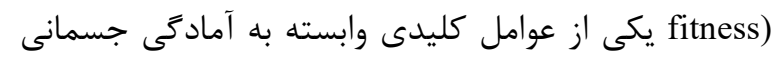
است كه با كاهش مركىومير و بيمارى إلهاى متابوليك همراه

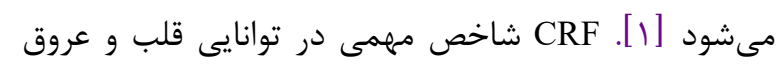

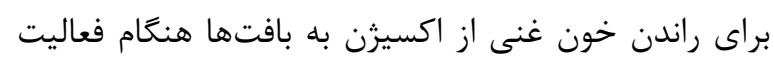

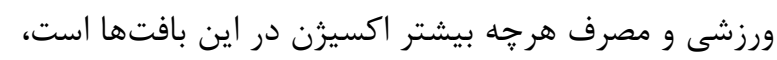

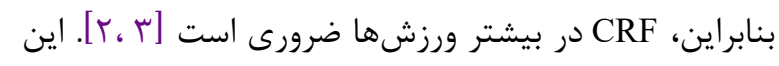

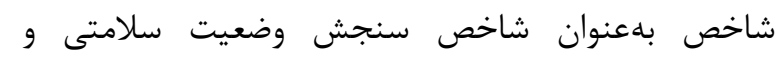

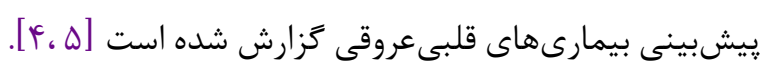

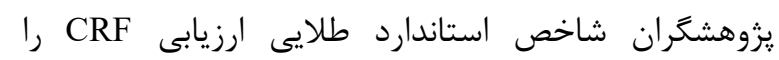


و اعتبارسنجى با روش معيار اجراى آزمونهاى ورزشى،

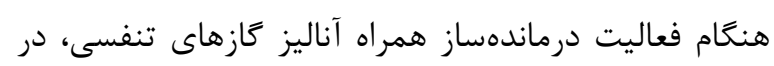

جمعيت يسران نوجوان بررسى معىشود.

\section{روش كار}

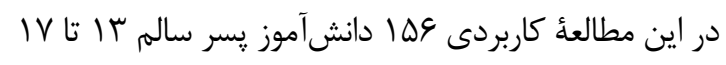
سال را از ميان مدارس مقطع تحصيلى متوسطه منتخب بلدروش ماري

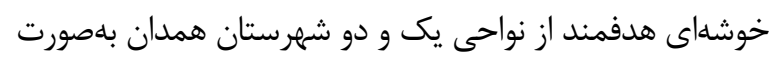
داوطلبانه انتخاب كرديم. ابتدا از دانشآموزان خواسته شد با مشورت والد والدين خود

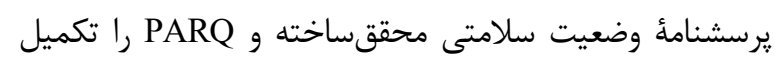

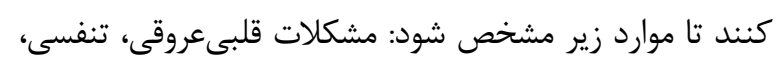

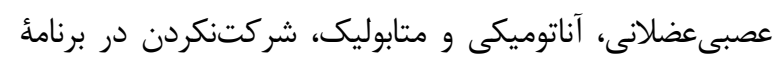

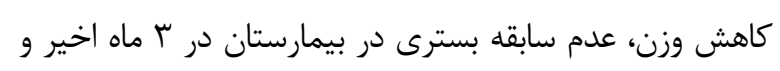

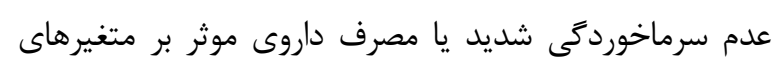

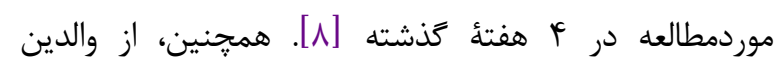

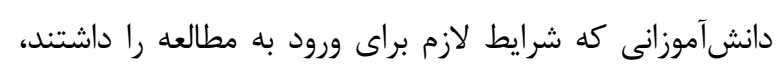

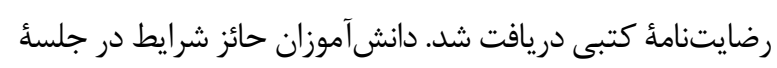

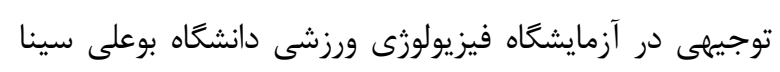

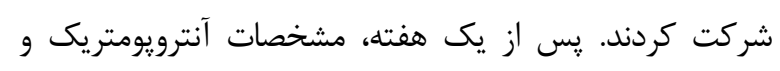

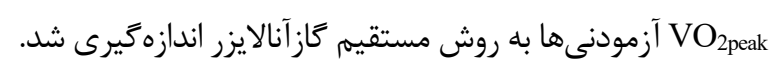

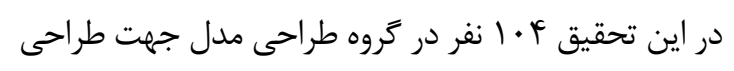

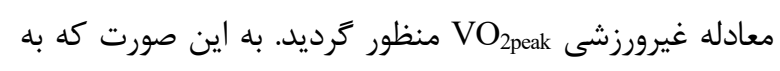

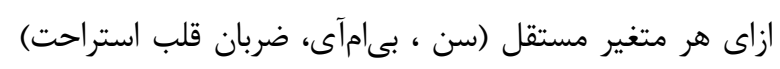

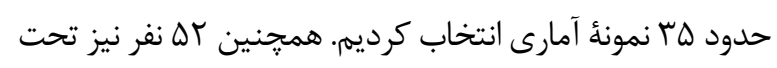

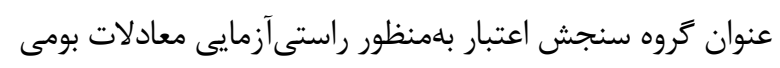

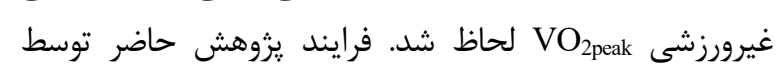

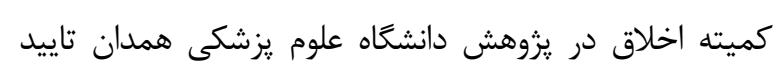

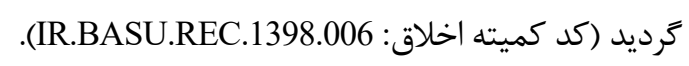
متغيرهاى سن (1/ • سال) ، قد باستفادهاز قدسنج ديوارى (IR.

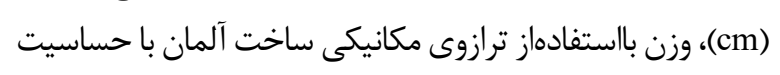

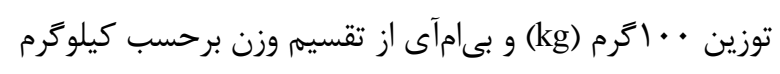

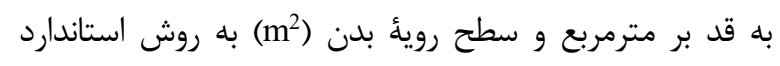

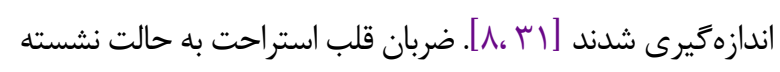

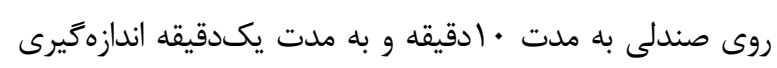

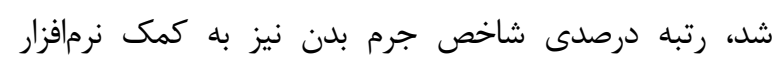
WHO_AnthroPlas_exe

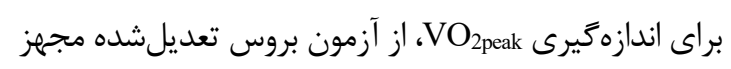
به دستخاه تجزيلوتحليل كازهاى تنفسى مختص كودكان استفاده

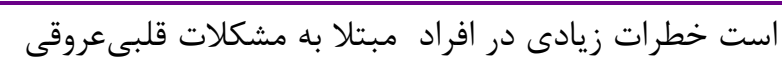

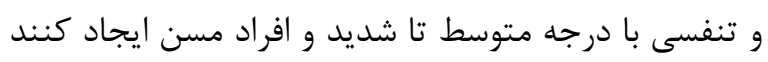

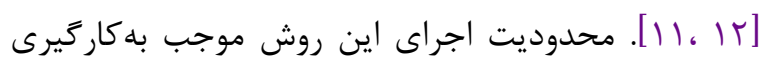

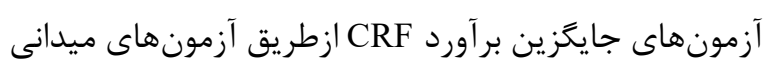

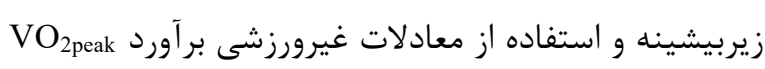

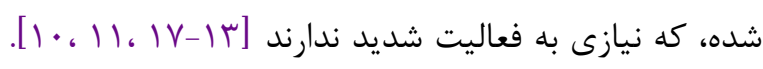

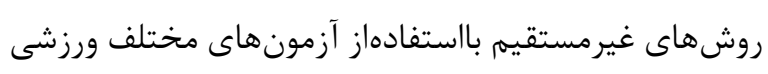

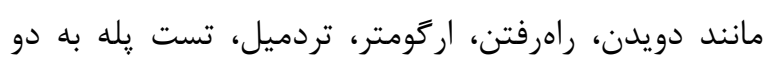

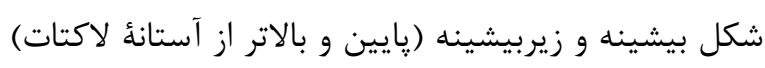

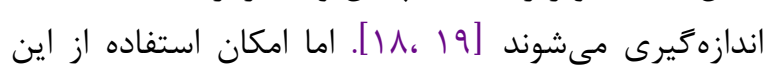

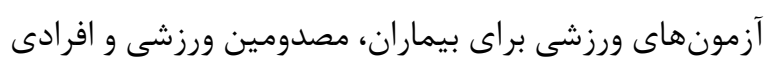

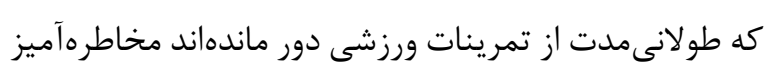

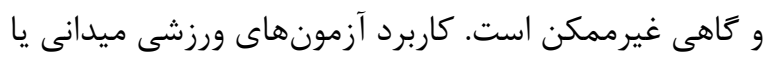

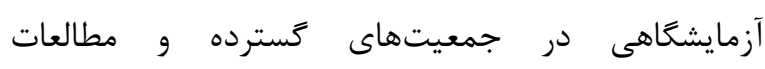

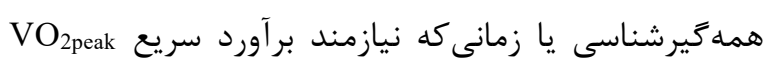

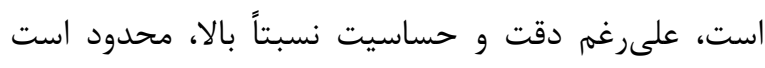

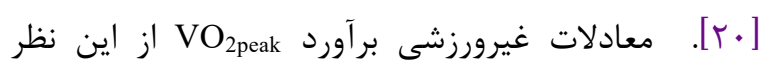

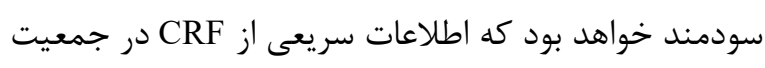

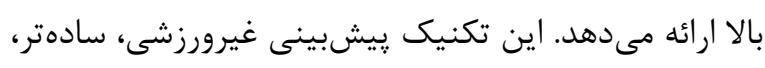

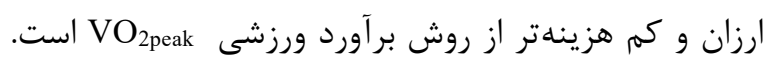

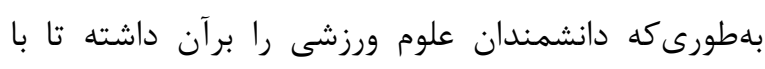

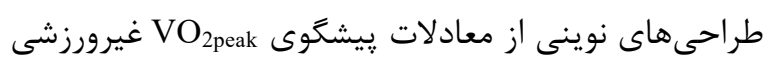

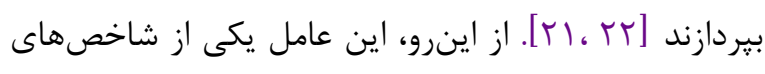

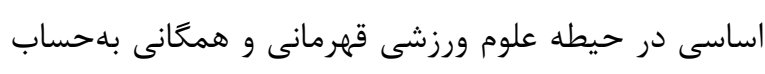

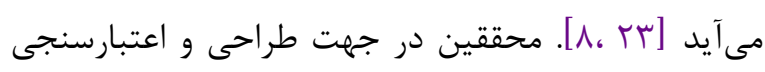

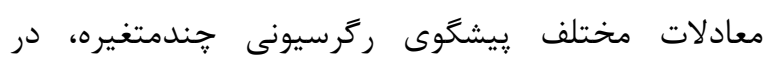

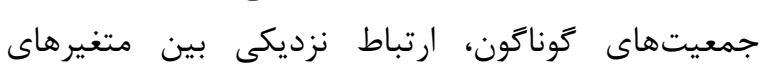

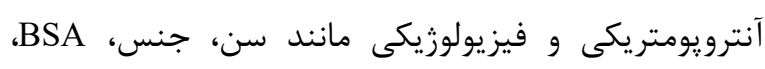

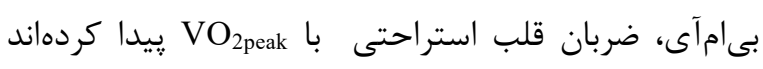

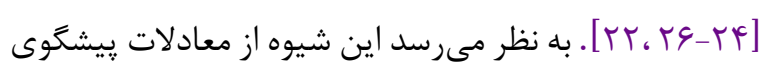

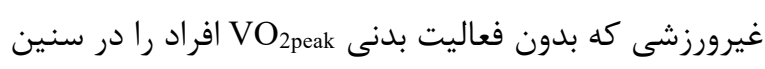

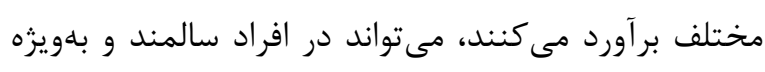

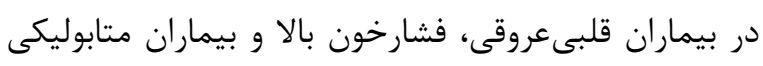

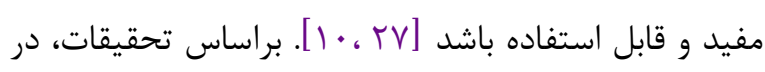

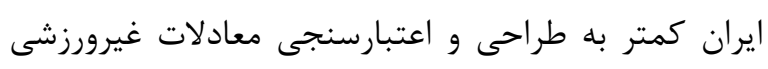

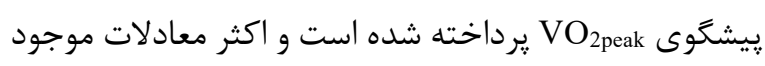

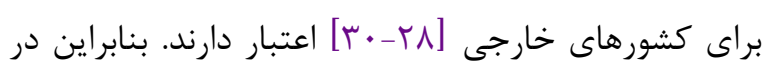

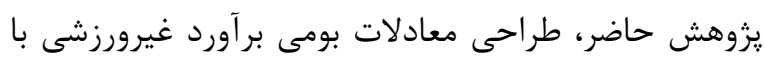

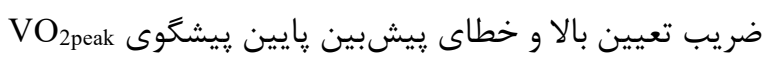


(GmbH, Germany

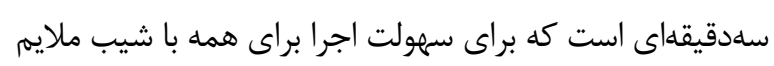
و سرعت كم شروع و بهتدريج افزايش مىيابد.
شد و روى تردميل الكتريكى مدل ( h/p/cosmos Saturn 300/125 Germany تنفسى PowerCube, Ganshorn Medizin Electronic)

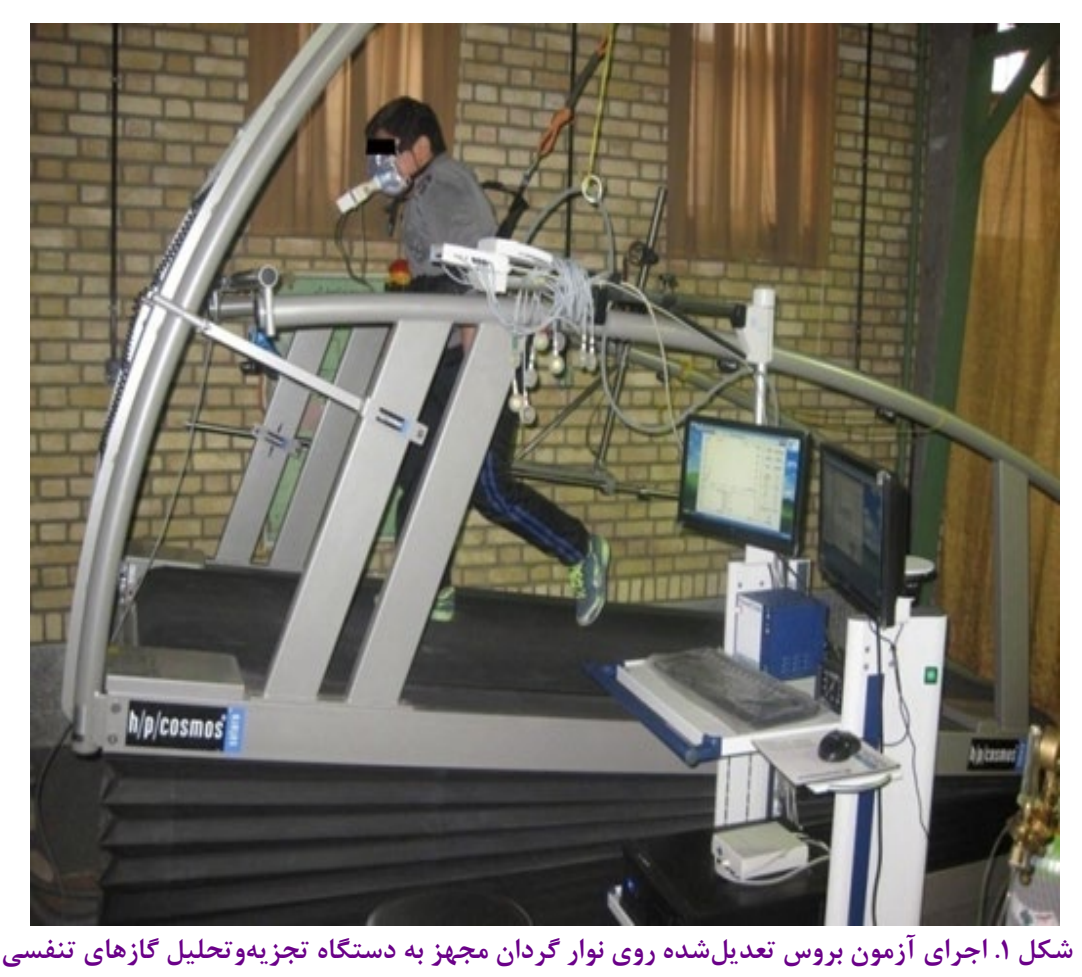

(Rudolph, Kansas City, MS, USA

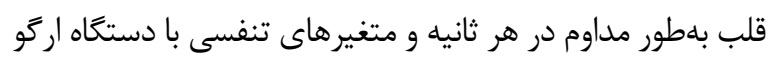

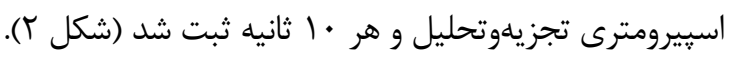

ضربان قلب و كازهاى تنفسى آزمودنىها در طول آزمون بلمترتيب با ضربان سنج يولار ( Polar Heart Rate Transmitter Hans (Model T34 Germany
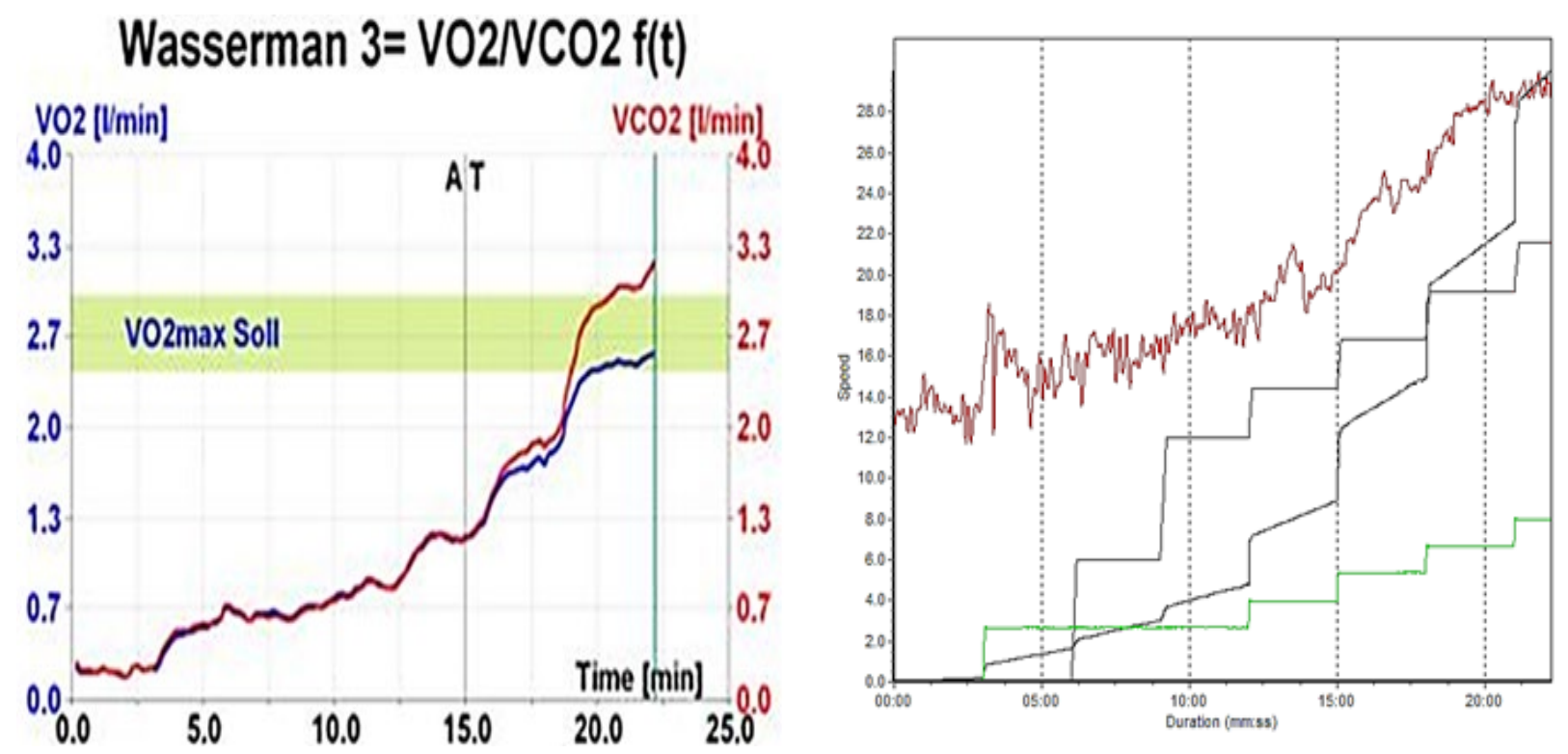

شكل r. تغييرات ضربان قلب و اكسيرن مصرفى فعاليت يك يسر 1 ساله در آزمون بروس تعديلشده 
همبستگى يُشسون استفاده شد. براى طراحى معادلئ برآورد

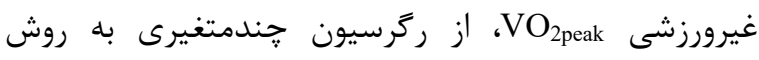
كامبه كام' (step-wise) براى طراحى مدل استفاده شد. ملاكى

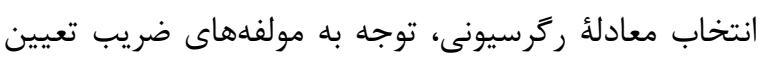

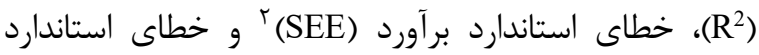

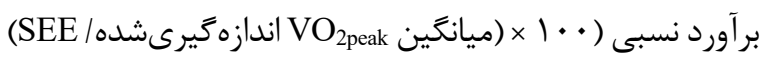

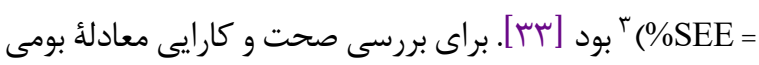

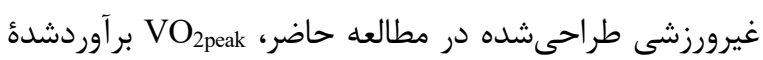

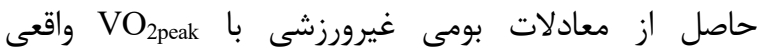

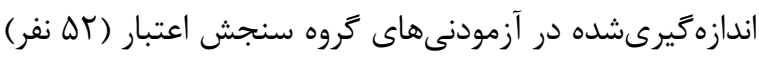

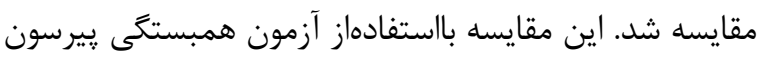

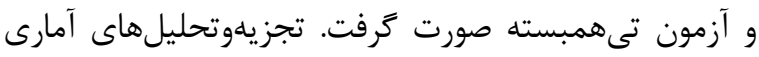

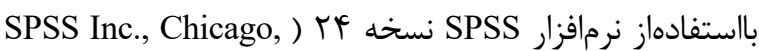
Ill., USA

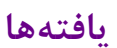

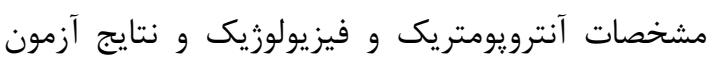
بروس تعديلشده دانشآموزان در جدول آ آمده است.

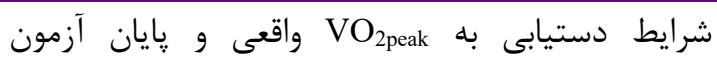
درماندهساز با حصول دو مورد از ملاكهاى زير محقق مى دشد؛

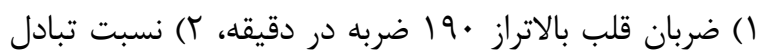

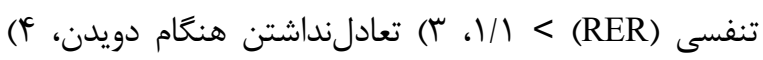

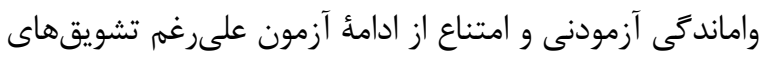

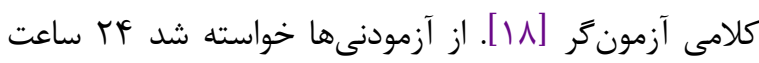

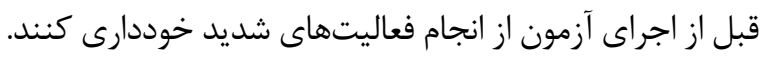

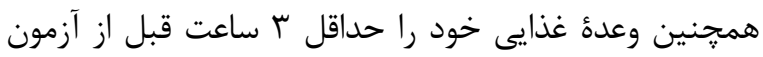

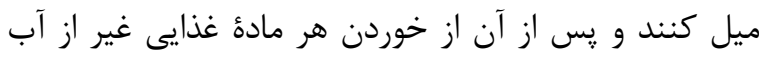

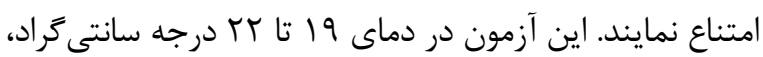

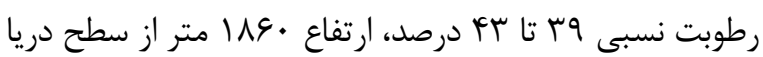
و با سطح آلايندههاى هوا در سطح نرمال در آزمايشكاه دانشكدة تربيت بدنى و علوم ورزشى دانشخاه بوعلى سينا اجرا شد.

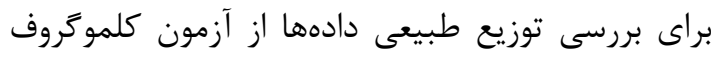
اسميرنوف (K-S) استفاده شد. از آمار يارامتريك و آمارههاى تراي

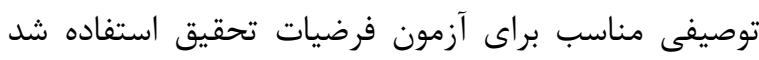

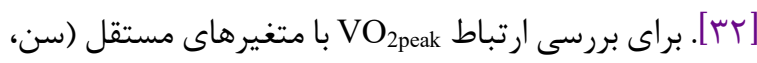

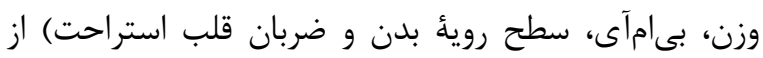

جدول 1. مشخصات آنتروفيزولوزيك يسران

\begin{tabular}{|c|c|c|c|}
\hline كرانهُ بالا & كرانهُ هايين & ميانَين \ انحراف معيار & متغير ها \\
\hline$I V / I V$ & $I T / V D$ & $\mid f / 1 \pm 94 / \pi r$ & سن (سال) \\
\hline 194 & 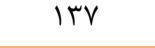 & $19 \mathrm{~V} / 9 \pm 9 \wedge / 99$ & قد (سانتىمتر ) \\
\hline 114 & $r \Lambda / \cdots$ & $\varphi \cdot \| \varphi \pm 14 / \cdot \varphi$ & وزن (كيلوَرم) \\
\hline$r \Delta / T V$ & $I T / F F$ & $r I / F \pm F r / \& q$ & بىامآى (كيلوكرم بر مترمربع) \\
\hline r/FFq & $1 / \cdot 91$ & $1 / \cdot \pm 9 V / T \varphi$ & رويئ سطح بدن (مترمربع) \\
\hline $1 \cdot r$ & $\Delta \Lambda$ & $\vee १ / ৭ \pm \Delta q / 9 \wedge$ & ضربان قلب استراحت (ضربه در دقيقه) \\
\hline 199 & 199 & $19 V / \cdot \pm \Delta F / 9 r$ & حداكثر ضربان قلب نظرى (ضربه در دقيقه) \\
\hline r/ve. & $\cdot / 9 \vee \cdot$ & $r / \cdot \pm F r \cdot \mid G T \Delta$ & بيشينهُ اكسيزن مصرفى مطلق (L/min) \\
\hline$\Delta F / \Lambda$. & $r t / \cdot$ & $|\varphi| / \varepsilon \pm 9 q / \& V$ & بيشينهُ اكسيزٌن مصرفى نسبى (ml/kg/min) \\
\hline $1 / \Delta \cdot$ & $1 / \cdot 9$ & $1 / \cdot \pm Y Y / \cdot \Lambda 1$ & نسبت تبادل تنفسى (RER) \\
\hline$F \mid / l$. & $\mid r / F \cdot$ & $T Y / Q \pm \cdot r / r \mid$ & اكسيرن مصرفى در آستانهٔ لاكتات (ml/kg/min) \\
\hline TrI & 19. & $r \cdot 1 / \cdot q \pm q / 1 r_{*}$ & ضربان قلب پايان آزمون فزاينده (ضربه در دقيقه) \\
\hline
\end{tabular}

معنادارى بين ميانگين VO2peak اندازهگيرىشده بلروش مستقيم و VO2peak برآورشده با معادلات خارجى منتخب مين

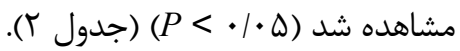

2 Standard Error of Estimate (SEE)

${ }^{3}$ SEE\% $=(S E E / H$ Rmax measured $) \times 100$

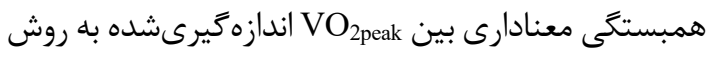
مستقيم با VO2peak برآوردشده از معادلات خارجى مشاهده

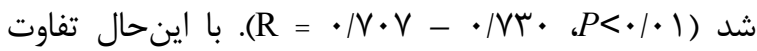


جدول r. ارزيابى صحت معادلات بر آورد غيرورزشى VO2peak خارجى در بِران ايرانى

\begin{tabular}{|c|c|c|c|c|}
\hline $\begin{array}{c}\text { Verma et al. (1986) } \\
\text { (५৭) }\end{array}$ & $\begin{array}{c}\text { Erdmann et al. } \\
(1999)\left(r_{\bullet}\right)\end{array}$ & $\begin{array}{l}\text { Bonen et al. } \\
(1979)(Y \wedge)\end{array}$ & 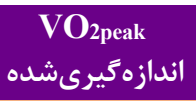 & معادله \\
\hline$r \cdot / \cdot \pm T r / \Delta T$ & $\Delta \cdot / 9 \pm 1 \mid / \varepsilon q$ & $\uparrow q / \Delta \pm q 9 / r \vee$ & $|F| / \varepsilon \pm q q / F V$ & $\mathrm{VO}_{2 \text { peak }}(\mathrm{ml} / \mathrm{kg} / \mathrm{min})$ \\
\hline$-1 / \varepsilon \pm F Y / T \varphi^{*}$ & $\Lambda / F \pm f / / \Lambda r^{*}$ & $N / f \pm r \cdot \mid q \varphi^{4}=$ & - & $\begin{array}{l}\text { ميانگين اختلاف } \\
\text { (ml/kg/min) }\end{array}$ \\
\hline •/VK・䋨 & $\cdot / V r \cdot$ 新 & $\cdot / V \cdot V$ 茟 & - & همبستگى \\
\hline
\end{tabular}

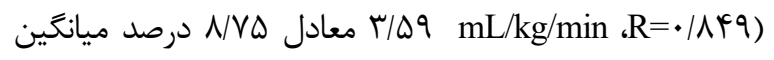
( ا ا تبيين مى كنند (ج) صحت معادلات غيرورزشى بومى برآورد

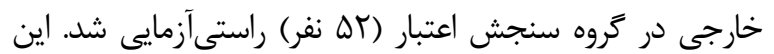

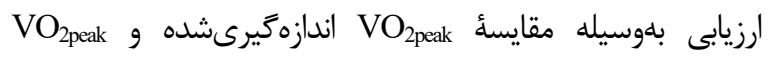
برآورشده به روش معادلات غيرورزشى صورت كرفت. نتايج همبستخى در دامنه بومى برآورد غيرورزشى VO2peak عملكرد بهترى درمقايسهبا معادلئ

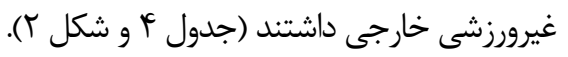

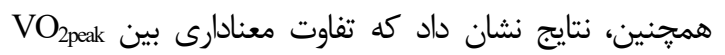

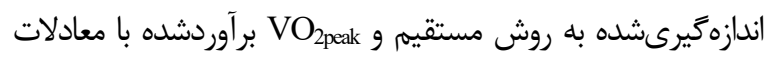

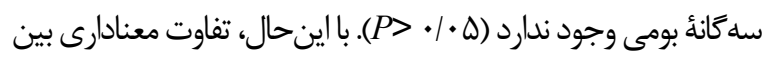
اندازهيرىشده به روش مستقيم و VO2peak معادلات غيرورزشى خارجى مشاهده شد (شكل س) (ه • / > > (P).

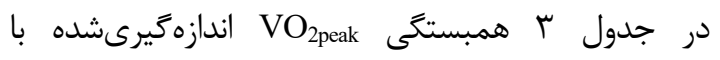
متغيرهاى مستقل (سن، وزن، بىامآى، BSA و جد

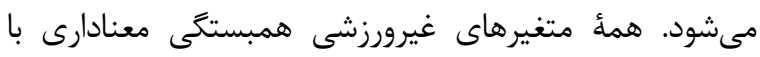

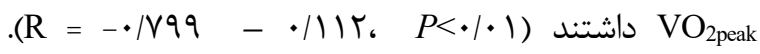

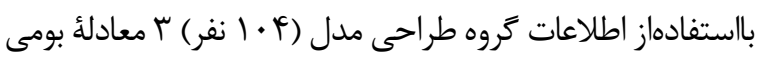

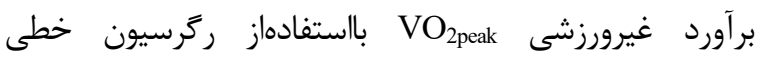
جندمتغيره بلروش step-wise استخراج شد (جدول \& ع). در معادله F

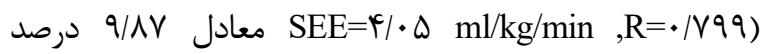
ميانكين (VO) را در مطالعهُ ما تبيين مى كند. در معادله F-

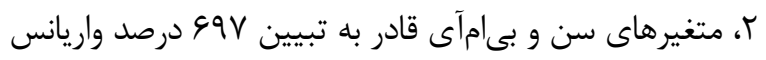

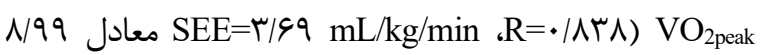

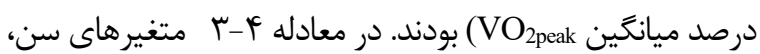

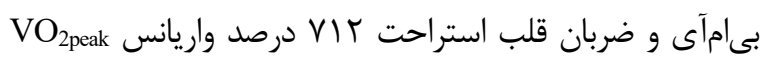

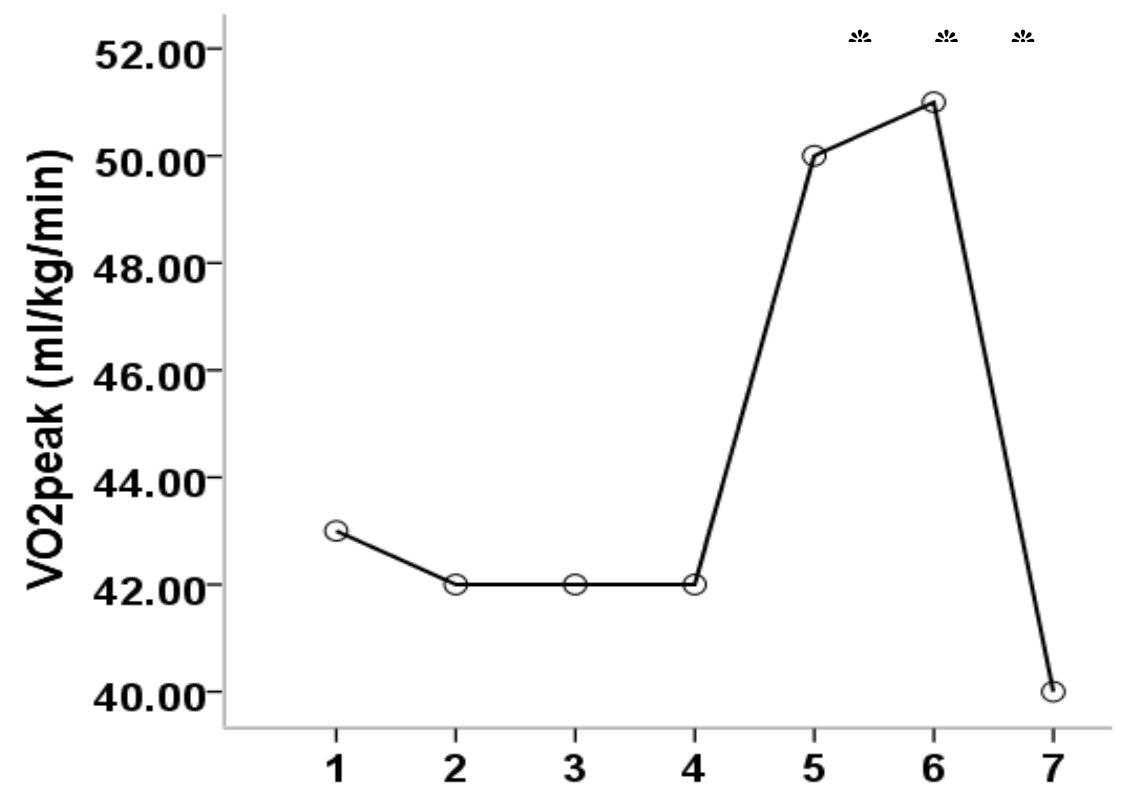

\section{Measured (1) and Predicted (2-7) VO2peak}

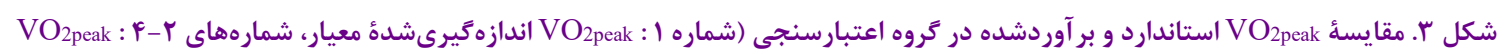

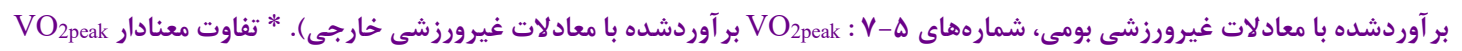

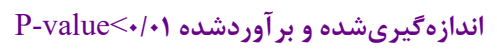




\begin{tabular}{|c|c|c|c|c|c|c|}
\hline $\mathbf{H R}_{\text {rest }}$ & BSA & BMI & 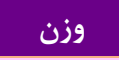 & سن & $\mathrm{VO}_{2 \text { peak }}$ & متغير ها \\
\hline$-\cdot / 49 V^{* * *}$ & $-.1849 *$ & $-\cdot / \vee 99 *$ & $-.1919^{*}$ & . IITt & 1 & $\mathrm{VO}_{2 \text { peak }}(\mathrm{mL} / \mathrm{kg} / \mathrm{min})$ \\
\hline 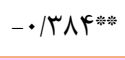 & $\cdot \mid$ FTI* & $\cdot / l v e$ & $\cdot /$ rat: & 1 & & سن (سال) \\
\hline$-\cdot / \cdot 14$ &.$/ 991 *$ & $\cdot|94|^{* * * m}$ & 1 & & & وزن (كيلوَرم) \\
\hline$\cdot / \cdot \Delta \Delta$ & $\cdot \mid \Lambda \Lambda Y^{*}$ & 1 & & & & BMI $\left(\mathrm{kg} / \mathrm{m}^{2}\right)$ \\
\hline$-\cdot / \cdot r \Delta$ & 1 & & & & & $\operatorname{BSA}\left(\mathbf{m}^{2}\right)$ \\
\hline 1 & & & & & & HRrest (bpm) \\
\hline
\end{tabular}

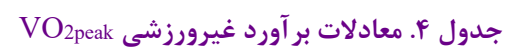

$\mathrm{VO}_{2 \text { peak }}(\mathrm{mL} / \mathrm{kg} / \mathrm{min})=9 F / r Y I-1 / \cdot \Delta \mathrm{V} \times \mathrm{BMI}\left(\mathrm{kg} / \mathrm{m}^{2}\right)$

$\left(\mathrm{R}^{2}=\cdot \mid\right.$ \& $\left.\mathrm{F} . \mathrm{SEE}=\boldsymbol{\varphi} / \cdot \Delta \mathrm{ml} / \mathrm{kg} / \mathrm{min}, P<\cdot / \cdot \cdot 1\right)$

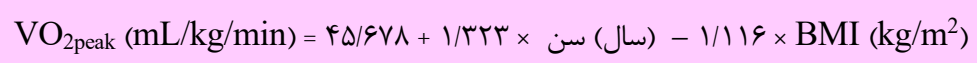
$\left.\left(\mathrm{R}^{2}=\cdot / 99 \mathrm{~V}, \mathrm{SEE}=r / 99 \mathrm{~mL} / \mathrm{kg} / \mathrm{min}, P<\cdot / \cdot \bullet\right)\right)$

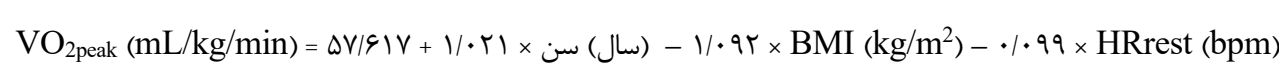
$\left.\left(\mathrm{R}^{2}=\cdot / \mathrm{V}\right) \mathrm{r} \cdot \mathrm{SEE}=r / \Delta 9 \mathrm{~mL} / \mathrm{kg} / \mathrm{min}, P<\cdot / \cdot \cdot 1\right)$
غعادلأ بر آورد

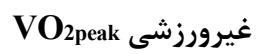

(1)

معادلهُ بر آورد

غيرورزشى مOى

(Y)

معادلة بر آورد

غيرورزشى معاد برورد

(r)
اختلاف ممكن است در سطح آمادگى، جنسيت و تغذئ آزمودنى ها باشد. ولى همبستخى معنادارى بين سن و VO

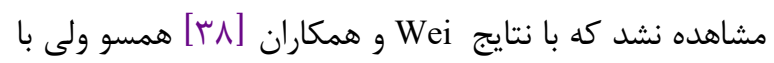

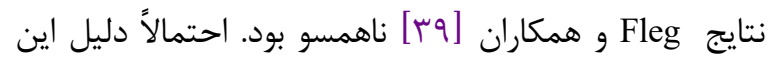
تفاوت، سن و دوران بلوغ آزمودنىها است. همجنين، صحت

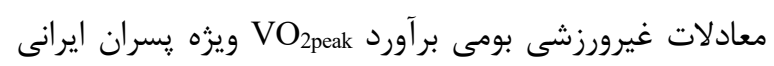

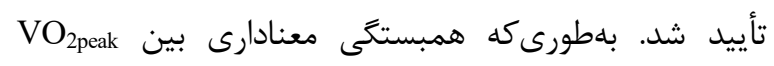

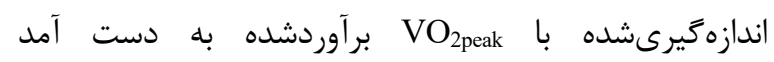

$$
\text { ( } \mathrm{R}=/ 4 \& 9-\cdot / \mathrm{V} \notin 9 \text { ، } P<\cdot 1 \cdot \cdot 1)
$$

VO يك معيار مطمئن براى ارزيابى CRFeak

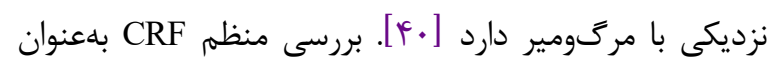
مهمترين شاخص سلامت عمومى براى افراد سالم و بيمار در بر برديى

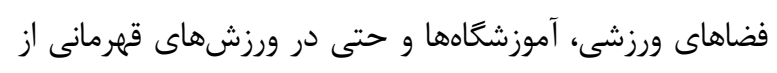

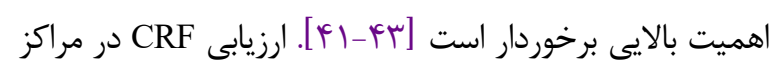
توانبخشى و درمانى، جهت محاسبة سطح كارايى دستخاه قلب و

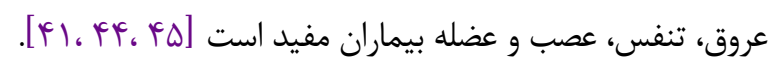

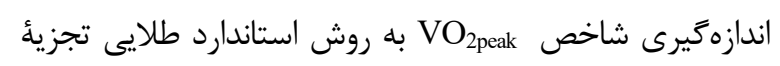

نتايج اين مطالعه نشان داد معادلات رگرسيونى غيرورزشى بومى در برآورد ظرفيت هوازى يسران ساتا لاساله از اعتبار

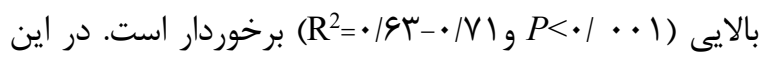

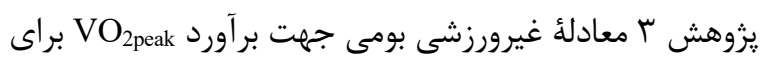

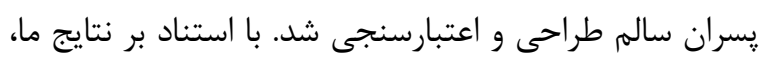

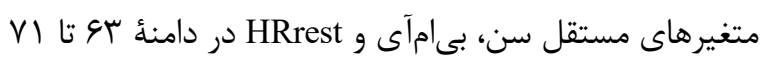

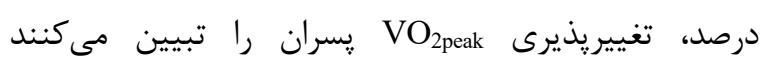

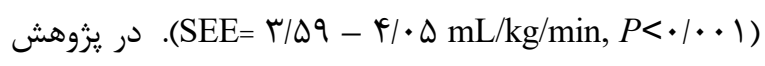
حاضر همبستخى بالايى بين بىامآى و VO2peak به دست آمد كه با نتايج Georgeو همكاران [19] و و [ها ] همسو بود. ولى Kind و همكاران [بr] تاثير دور كمر بر راقوىتر از بىامآى در مردان كزارش كرد. دليل تفاوت با يافتههاى تحقيق حاضر احتمالاً بهخاطر تأثير بالاى جاقى

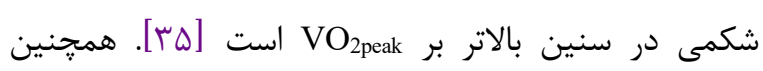

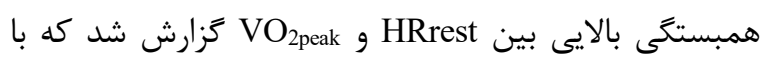

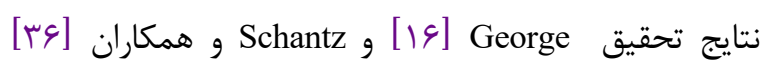
همسو ولى با نتايج تحقيق صالحى [rV] ناهمسو بود. اين 


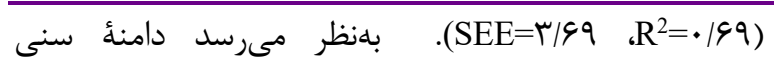

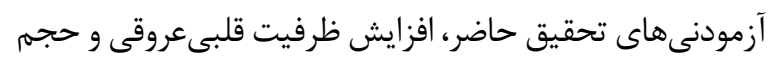
عضلانى در بالابردن Vo2peak ناشى از سن تأثير خذار باشد.

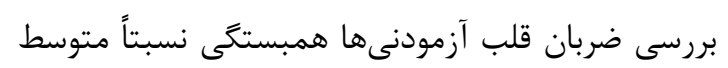

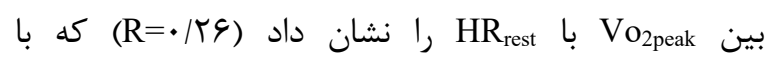
يزوهشهاى قبلى در اين زمينه همسو است. در تحقيقات مختلف تغييرات مقدار همبستخى متغير مستقل Vo 2peak

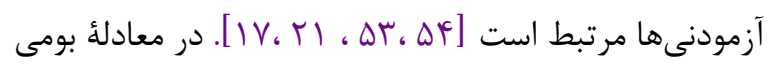

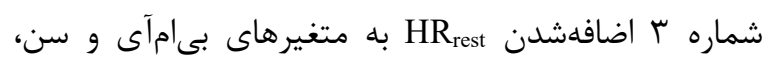
بهطور معنادارى ضريب تعيين را بالا مىبرد و خطاى استاندارد

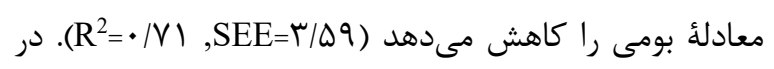

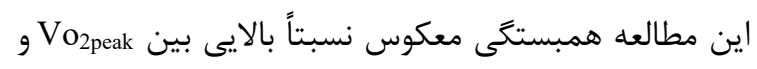
BSA

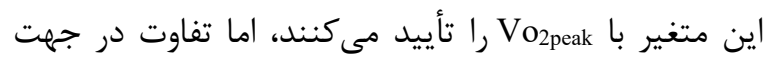
همبستخى ممكن است به تغييرات فيزيولوزيكى در سن بلوغ

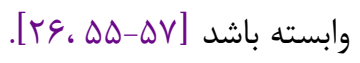
يافتههاى ما نشان مىدهد استفاده از بيش از يك متغير

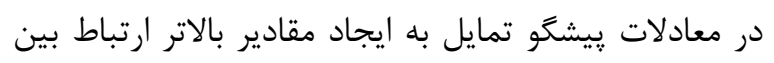

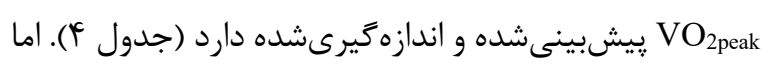
بررسى مؤلفههاى همخطى در الگوى رگرسيون زيندمتغيره

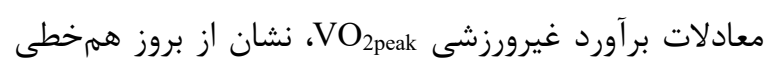

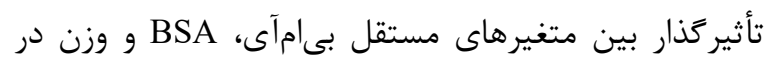
معادلات بود كه باعث حذف اين متغيرها از معادلات بومى اين

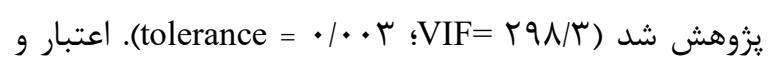

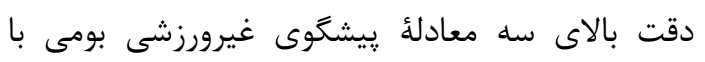

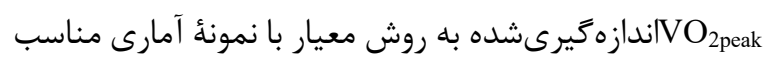

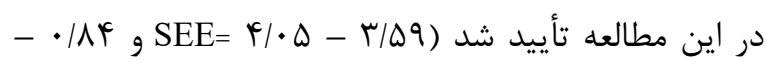
فئ (R= • /V9

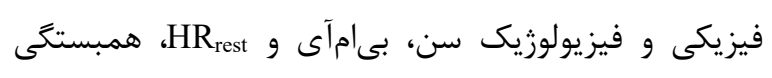

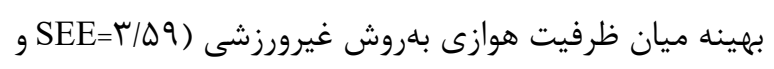

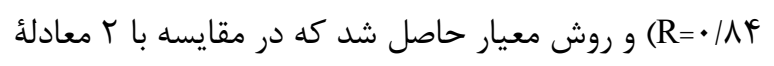

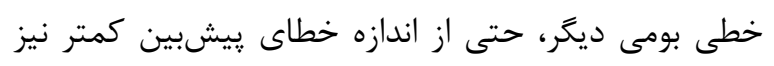
برخوردار بود.

\section{نتيجه كيرى}

در تحقيق حاضر عدم مداخله تغذيهاى آزمودنىها و استفاده از فقط جنسيت يسر از محدوديتهاى تحقيق حاضر بود. باتوجه به اينكه مطالعات محدودى در برآورد غيرورزشى VO2peak در سنين نوجوانى،
كازهاى تنفسى [عץ] در نمونههاى بزرك انسانى يا مطالعات

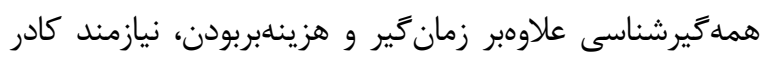

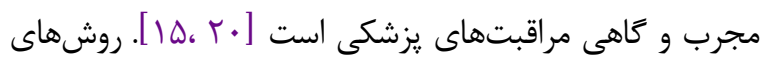
اندازمخيرى غيرمستقيم VO

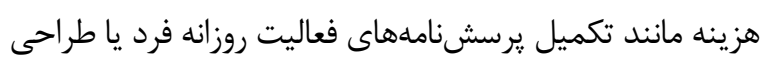

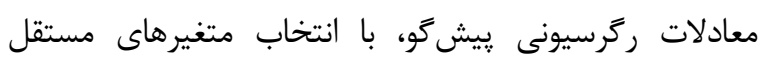

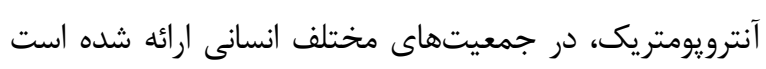

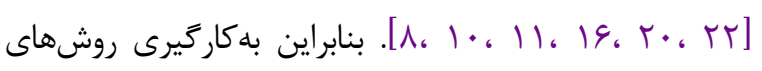

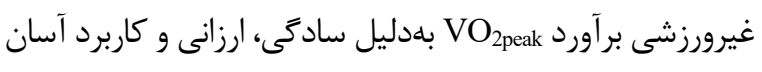

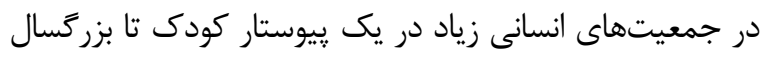

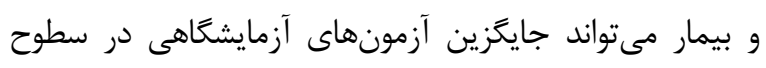

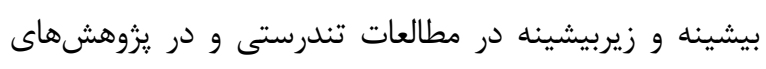
غربال زرى شود.

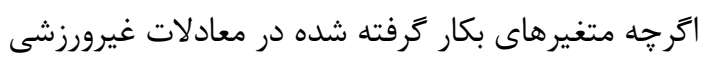

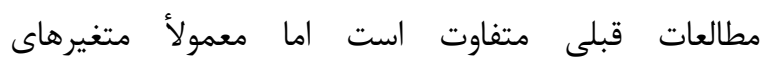
آنترويومتريكى، سن، ضربان قلب در زمان استراحت و فعاليت،

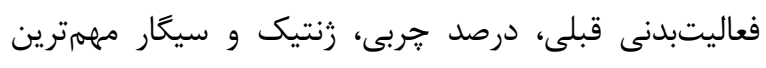

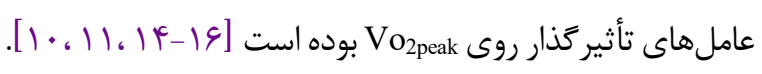

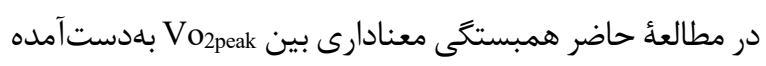

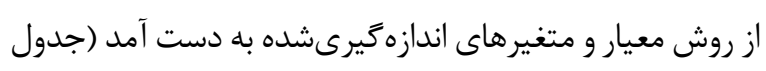

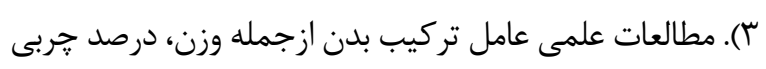

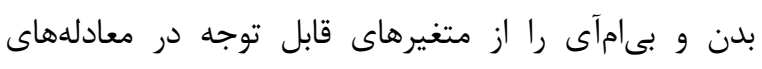
ركر سيونى خطى و غيرخطى بر آورد

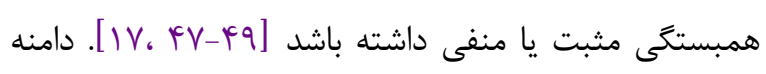

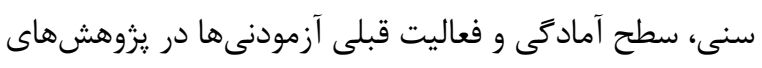

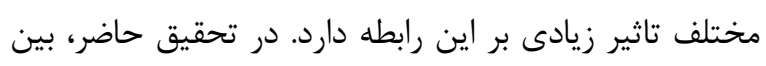

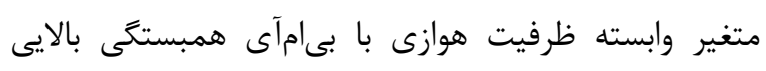

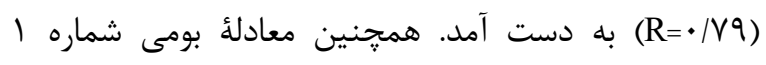

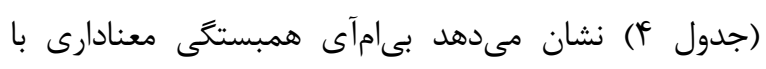

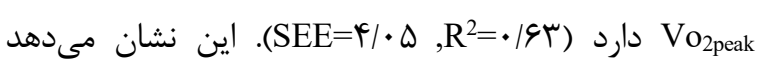

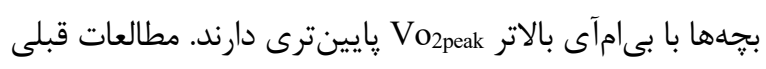
نشان مىدهد Vo

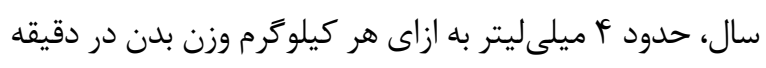

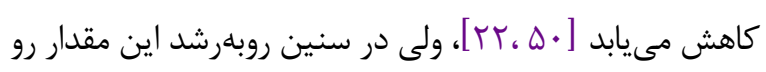

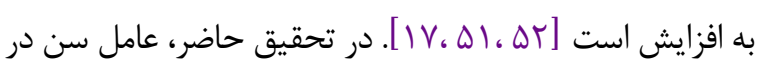

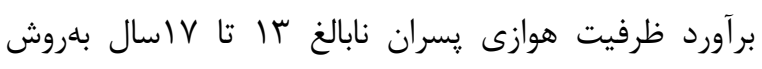

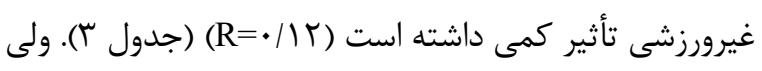

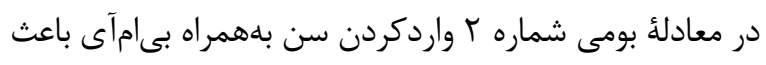

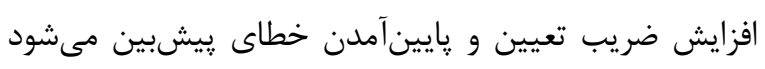




$$
\begin{aligned}
& \text { فرزاد ناظه و همكاران ا برآورد غيرورزشى ظرفيت هوازى }
\end{aligned}
$$

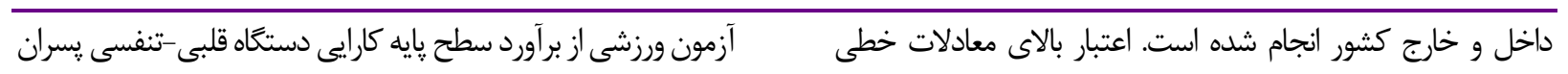

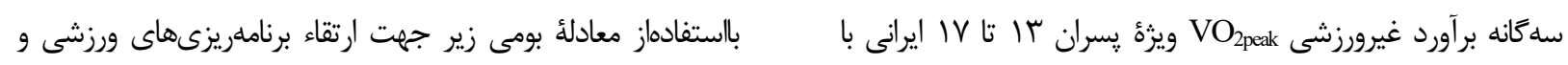

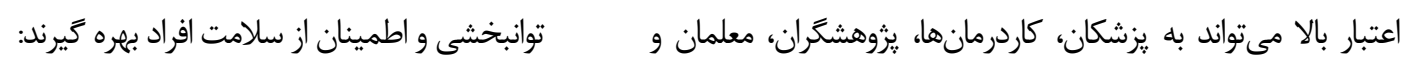

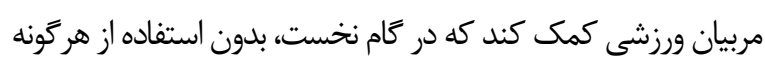

$$
\begin{aligned}
& \mathrm{VO}_{2 \text { peak }}(\mathrm{mL} / \mathrm{kg} / \mathrm{min})=\Delta \mathrm{V} / 9|\mathrm{~V}+1 / \cdot r| \times \text { سال(س) }-1 / \cdot 9 r \times \mathrm{BMI}\left(\mathrm{kg} / \mathrm{m}^{2}\right)-\cdot 1 \cdot 99 \times \text { Hrrest (bpm) } \\
& \text { تعارض منافع } \\
& \text { بين نويسند ًان هيجَّونه تعارضى در منافع وجود ندارد. } \\
& \text { منابع مالى } \\
& \text { اين مطالعه از اعتبار يزوهانه هيئت علمى حوزه معاونت } \\
& \text { يزوهش و فناورى دانشكاه بوعلى سينا انجام كرفت. } \\
& \text { تقدير و تشكر }
\end{aligned}
$$

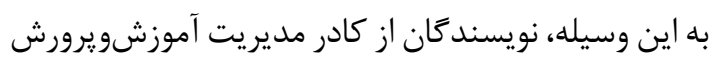

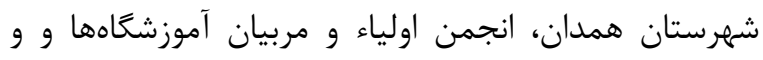

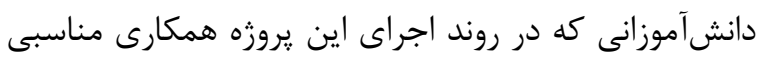

$$
\begin{aligned}
& \text { داشتند، تشكر مىنمايند. }
\end{aligned}
$$

\section{References}

1. Booth FW, Roberts CK, Laye MJ. Lack of exercise is a major cause of chronic diseases. Compr Physiol. 2012; 2(2):1143-1211. [DOI:10.1002/cphy.c110025] [PMID] [PMCID]

2. Lavie CJ, Arena R, Swift DL, Johannsen, NM, Sui X, Lee DC, Earnest CP. Exercise and the cardiovascular system: clinical science and cardiovascular outcomes. Circ Res. 2015; 117(2):207-19. [DOI:10.1161/CIRCRESAHA.117.305205] [PMID] [PMCID]

3. Reis JF, Fatela P, Mendonca GV, Vaz JR, Valamatos MJ, Infante J, et al. Tissue Oxygenation in Response to Different Relative Levels of Blood-Flow Restricted Exercise. Front Physiol. 2019; 10:407. [DOI:10.3389/fphys.2019.00407] [PMID] [PMCID]

4. Kodama S, Saito K, Tanaka S, Maki M, Yachi Y, Asumi $\mathrm{M}$, et al. Cardiorespiratory fitness as a quantitative predictor of all-cause mortality and cardiovascular events in healthy men and women: a meta-analysis. JAMA. 2009; 301(19):2024-35. [DOI:10.1001/jama.2009.681] [PMID]

5. Prieto-Benavides DH, García-Hermoso A, Izquierdo M, Alonso-Martínez AM, Agostinis-Sobrinho C, Correa-Bautista JE, et al. Cardiorespiratory Fitness Cut-Points are Related to Body Adiposity Parameters in Latin American Adolescents. Medicina. 2019; 55(9):508. [PMID] [PMCID]

6. Rasch-Halvorsen $\varnothing$, Hassel E, Langhammer A, Brumpton BM, Steinshamn S. The association between dynamic lung volume and peak oxygen uptake in a healthy general population: the HUNT study. BMC Pulmon Med. 2019; 19(1):2. [DOI:10.1186/s12890-018-0762-x] [PMID] [PMCID]

7. Poole DC, Jones AM. Measurement of the maximum oxygen uptake VO2max: VO2peak is no longer acceptable. J App Phys. 2017;122(4):997-1002. [DOI:10.1152/japplphysiol.01063.2016] [PMID]
8. American College of Sports Medicine, editor. ACSM's health-related physical fitness assessment manual. Philadelphia: Lippincott Williams \& Wilkins; 2013.

9. Cade WT, Bohnert KL, Reeds DN, Peterson LR, Bittel AJ, Bashir A, Byrne BJ, Taylor CL. Peak oxygen uptake (VO2peak) across childhood, adolescence and young adulthood in Barth syndrome: Data from cross-sectional and longitudinal studies. PloS One. 2018;13(5):e0197776. [DOI:10.1371/journal.pone.0197776] [PMID] [PMCID]

10. Jurca R, Jackson AS, LaMonte MJ, Morrow JR, Blair $\mathrm{SN}$, Wareham NJ, et al. Assessing cardiorespiratory fitness without performing exercise testing. America J Prev Med. 2005; 29(3):185-93. [DOI:10.1016/j.amepre.2005.06.004] [PMID]

11. Shenoy S, Tyagi BS, Sandhu JS. Concurrent validity of the non-exercise-based VO2max prediction equation using percentage body fat as a variable in Asian Indian adults. Sports Med, Arthroscopy, Rehab, Ther Tech. 2012; 4(1):34. [DOI:10.1186/1758-2555-4-34] [PMID] [PMCID]

12. Sartor F, Vernillo G, De Morree HM, Bonomi AG, La Torre A, Kubis HP, Veicsteinas A. Estimation of maximal oxygen uptake via submaximal exercise testing in sports, clinical, and home settings. Sports Med. 2013; 43(9):865-73. [DOI:10.1007/s40279013-0068-3] [PMID]

13. St Clair Gibson A, Broomhead S, Lambert MI, Hawley JA. Prediction of maximal oxygen uptake from a $20-\mathrm{m}$ shuttle run as measured directly in runners and squash players. Journal of Sports Sciences. 1998 Jan 1;16(4):331-5. [DOI:10.1080/02640419808559361] [PMID]

14. Bradshaw DI, George JD, Hyde A, LaMonte MJ, Vehrs PR, Hager RL, Yanowitz FG. An accurate VO2max nonexercise regression model for 18-65year-old adults. Res Quart Exercise Sport. 2005; 
76(4):426-32.

[DOI:10.1080/02701367.2005.10599315] [PMID]

15. Schembre SM, Riebe DA. Non-exercise estimation of VO2max using the international physical activity questionnaire. Meas Phys Edu Exer Sci. 2011; 15(3):168-81.

[DOI:10.1080/1091367X.2011.568369] [PMID] [PMCID]

16. George JD, Paul SL, Hyde A, Bradshaw DI, Vehrs PR, Hager RL, Yanowitz FG. Prediction of maximum oxygen uptake using both exercise and non-exercise data. Meas Phys Edu Exer Sci. 2009; 13(1):1-2. [DOI:10.1080/10913670802609086]

17. Rexhepi AM, Brestovci B. Prediction of VO2max based on age, body mass, and resting heart rate. Human Mov. 2014; 15(1):56-9. [DOI:10.2478/humo2014-0003]

18. Klusiewicz A, Borkowski L, Sitkowski D, BurkhardJagodzińska K, Szczepańska B, Ladyga M. Indirect methods of assessing maximal oxygen uptake in Rowers: Practical implications for evaluating physical fitness in a training cycle. J Human Kinetics. 2016; 50(1):187-94. [DOI:10.1515/hukin-20150155] [PMID] [PMCID]

19. Davis JA. Direct determination of aerobic power. Phys Assess Human Fit. 1995:9-17.

20. Ramsbottom R, Brewer J, Williams C. A progressive shuttle run test to estimate maximal oxygen uptake. British J Sports Med. 1988; 22(4):141-4. [DOI:10.1136/bjsm.22.4.141] [MID] [MCID]

21. Kind S, Brighenti-Zogg S, Mundwiler J, Schüpbach U, Leuppi JD, Miedinger D, Dieterle T. Factors Associated with Cardiorespiratory Fitness in a Swiss Working Population. J Sports Med. 2019; Article ID: 5317961. [DOI:10.1155/2019/5317961] [PMID] [PMCID]

22. Neto M, de Albuquerque G, Farinatti PD. Nonexercise models for prediction of aerobic fitness and applicability on epidemiological studies: descriptive review and analysis of the studies. Revista Brasileira de Medicina do Esporte. 2003; 9(5):304-14. [DOI:10.1590/S1517-86922003000500006]

23. Loe H, Nes BM, Wisløff U. Predicting VO2peak from submaximal-and peak exercise models: the HUNT 3 fitness study, Norway. PloS One. 2016; 11(1): 0144873.

[DOI:10.1371/journal.pone.0144873] [PMID] [PMCID]

24. Zhang T, Zhang CF, Jin F, Wang L. Association between genetic factor and physical performance. Hereditas. 2004; 26(2):219-26.

25. Hirai T, Kusaka Y, Suganuma N, Seo A, Tobita Y. Work form affects maximum oxygen uptake for one year in workers. Indust Health. 2011; 49(3):321-7. [DOI:10.2486/indhealth.MS870] [PMID]

26. Chatterjee S, Chatterjee P. Prediction of maximal oxygen consumption from body mass, height and body surface area. Indian J Physiol Pharmacol. 2006; 50(2):181-6.
27. Rao AV, Phadke AV, Patil PB, Joshi AR. Comparison of non-exercise test and step test in estimation of aerobic capacity (VO2max) in young adults. National J Phys Pharm Pharmac. 2014; 4(3):218. [DOI:10.5455/nippp.2014.4.150420141]

28. Bonen A, Heyward VH, Cureton KJ, Boileau RA, Massey BH. Prediction of maximal oxygen uptake in boys, ages 7-15 years. Med Sci Sport. 1979; 11(1):249.

29. Verma SS, Gupta RK, Kishore N, Sen GJ. A simple relationship between maximal aerobic power and body weight in Indian adolescent boys. India J Med Sci. 1986; 40(4):93.

30. Erdmann LD, Hensley LD, Dolgener FA, Graham RE. Nonexercise Prediction of VO2peak in Middle School-Age Boys. Meas Phys Educ Exer Sci. 1999; 3(1):37-50. [DOI:10.1207/s15327841mpee0301_3]

31. Haycock GB, Schwartz GJ, Wisotsky DH. Geometric method for measuring body surface area: a heightweight formula validated in infants, children, and adults. J Ped. 1978; 93(1):62-6. [DOI:10.1016/S0022-3476(78)80601-5]

32. Hinkle DE, Wiersma W, Jurs SG. Applied statistics for the behavioral sciences. Massachusetts: Houghton Mifflin; 2003.

33. Montgomery DC, Peck EA, Vining GG. Introduction to linear regression analysis. Washington D.C.: John Wiley \& Sons; 2012.

34. Kind S, Brighenti-Zogg S, Mundwiler J, Schüpbach U, Leuppi JD, Miedinger D, Dieterle T. Factors Associated with Cardiorespiratory Fitness in a Swiss Working Population. J Sport Med. 2019; Article ID: 5317961. [DOI:10.1155/2019/5317961] [PMID] [PMCID]

35. Dagan SS, Segev S, Novikov I, Dankner R. Waist circumference vs body mass index in association with cardiorespiratory fitness in healthy men and women: a cross sectional analysis of 403 subjects. Nut J. 2013; 12(1):12. [DOI:10.1186/1475-2891-12-12] [ㅁID] [PMCID]

36. Schantz P, Eriksson JS, Rosdahl $\mathrm{H}$. The heart rate method for estimating oxygen uptake: analyses of reproducibility using a range of heart rates from commuter walking. Eur J App Phys. 2019; 119(1112):2655-71. [DOI:10.1007/s00421-019-04236-0] [PMID] [PMCID]

37. Salehi S, Poursaeid Esfahani M, Hassabi M. Determine the estimated validity of Vo2max based on the formula in the female athlete and non-athlete. Res Med. 2020; 44(1): 326-30.

38. Wei Z, Chen L, Hou X, van Zijl PC, Xu J, Lu H. Agerelated alterations in brain perfusion, venous oxygenation, and oxygen metabolic rate of mice: a 17-month longitudinal MRI study. Front Neurol. 2020; 11. [DOI:10.3389/fneur.2020.00559] [PMID] [PMCID]

39. Fleg JL, Morrell CH, Bos AG, Brant LJ, Talbot LA, Wright JG, Lakatta EG. Accelerated longitudinal decline of aerobic capacity in healthy older adults. $\begin{array}{lll}\text { Circul. } & \text { 2005; }\end{array}$ 
[DOI:10.1161/CIRCULATIONAHA.105.545459] [PMID]

40. Castillo-Garzón MJ, Ruiz JR, Ortega FB, Gutiérrez Á. Anti-aging therapy through fitness enhancement. Clin Intervent Age. 2006; 1(3):213. [DOI:10.2147/ciia.2006.1.3.213] [PMID] [PMCID]

41. Dwyer GB, Davis MD. ACSM's health related physical fitness manual. New Jersey: Lippincott Williams \& Wilkins; 2008.

42. Solway S, Brooks D, Lacasse Y, Thomas S. A qualitative systematic overview of the measurement properties of functional walk tests used in the cardiorespiratory domain. Chest. 2001; 119(1):25670. [DOI:10.1378/chest.119.1.256] [PMID]

43. Mackenzie B. Performance evaluation tests. London: Electric World Plc. 2005; 24(25):57-158.

44. Boreham C, Twisk J, Neville C, Savage M, Murray L, Gallagher A. Associations between physical fitness and activity patterns during adolescence and cardiovascular risk factors in young adulthood: the Northern Ireland Young Hearts Project. Int J Sports Med. 2002; 23(S1):22-6. [DOI:10.1055/s-200228457] [PMID]

45. Anderssen SA, Cooper AR, Riddoch C, Sardinha LB, Harro M, Brage S, Andersen LB. Low cardiorespiratory fitness is a strong predictor for clustering of cardiovascular disease risk factors in children independent of country, age and sex. Europ J Cardiovasc Prev Rehab. 2007; 14(4):526-31. [DOI:10.1097/HJR.0b013e328011efc1] [PMID]

46. Wasserman K. Diagnosing cardiovascular and lung pathophysiology from exercise gas exchange. Chest. 1997; [DOI:10.1378/chest.112.4.1091] [PMID]

47. Sloan RA, Haaland BA, Leung C, Padmanabhan U, Koh HC, Zee A. Cross-validation of a non-exercise measure for cardiorespiratory fitness in Singaporean adults. Singapore Med J. 2013; 54(10):576-80. [DOI:10.11622/smedj.2013186] [PMID]

48. Sharma M, Kamal R, Chawla K. Correlation of body composition to aerobic capacity; A cross sectional study. Int J App Res. 2016; 2(1):38-42.

49. Wier LT, Jackson AS, Ayers GW, Arenare B. Nonexercise models for estimating VO2max with waist girth, percent fat, or BMI. Med Sci Sports Exer. 2006; 38(3):555-61. [DOI:10.1249/01.mss.0000193561.64152] [PMID]

50. Hale T. Exercise physiology: a thematic approach. New Jersey: John Wiley \& Sons; 2005.

51. Menezes Júnior FJ, Jesus ÍC, Leite N. Predictive equations of maximum oxygen consumption by shuttle run test in children and adolescents: A systematic review. Revista Paulista de Pediatria. 2019: 37(2). [DOI:10.1590/19840462/;2019;37;2;00016] [PMID] [PMCID]

52. Armstrong N, Barker AR. Oxygen uptake kinetics in children and adolescents: a review. Pediat Exer Sci. 2009; 21(2):130-47. [DOI:10.1123/pes.21.2.130] [PMID]
53. Nazem F, Saki S, Jalili M. Validation of Francis Step Protocol by Respiratory Gases Analyses and Design Native Equation to Estimate Aerobic Capacity in Iranian Boys. Knowledge Health. 2017; 12(1):66-72.

54. Uth N, Sørensen H, Overgaard K, Pedersen PK. Estimation of $\dot{\mathrm{VO}} 2 \mathrm{max}$ from the ratio between HR max and HR rest-the Heart Rate Ratio Method. Euro J App Phys. 2005; 91(1):111-5. [DOI:10.1007/s00421-003-0988-y] [PMID]

55. Narang N, Thibodeau JT, Levine BD, Gore MO, Ayers CR, Lange RA, et al. Inaccuracy of estimated resting oxygen uptake in the clinical setting. Circul. 2014; 129(2):203-10 [DOI:10.1161/CIRCULATIONAHA.113.003334] [PMID]

56. Tonelli AR, Wang XF, Abbay A, Zhang Q, Ramos J, McCarthy K. Can we better estimate resting oxygen consumption by incorporating arterial blood gases and spirometric determinations? Respir Care. 2015; 60(4):517-25. [DOI:10.4187/respcare.03555] [PMID] [PMCID]

57. Shephard RJ, Weese CH, Merriman JE. Prediction of maximal oxygen intake from anthropometric data. Int J App Phys Inc Occ Phys. 1971; 29(2):119-30. [DOI:10.1007/BF00698022] [PMID] 